\title{
MACROVASOS CONGÉNITOS RETINIANOS: \\ UN HALLAZGO CASUAL
}

\section{CONGENITAL RETINAL MACROVESSELS: A DISCOVERY BY CHANCE}

\author{
PÉREZ-CARRO G ${ }^{1}$, MIRANDA-ROLLÓN Mํ, JUNCEDA-MORENO C ${ }^{1}$, ÁLVAREZ-SÁNCHEZ M \\ JUNCEDA-MORENO J²
}

\section{RESUMEN}

Caso clínico: Los macrovasos congénitos retinianos son raros, son ramas aberrantes, vénulas en su mayoría, localizadas en el polo posterior que de forma típica cruzan la zona avascular foveal.

Discusión: En la mayoría de los casos son lesiones unilaterales y estables, sin repercusión visual por lo que su diagnóstico es casual. En ocasiones se asocian a localizaciones atípicas de la fóvea, quistes foveales e incluso hemorragias. Por lo general debe realizarse un diagnóstico diferencial con otras entidades vasculares retinianas como el hemangioma capilar, angioma racemoso e incluso si se acompaña de síntomas neurológicos descartar el síndrome de Wyburn-Masson.

Palabras clave: Macrovasos congénitos retinianosvasos retina aberrantes-ectopia foveal.

\begin{abstract}
Clinical case: Congenital retinal macrovessels, where an aberrant retinal vessel, usually a venule, is present in the posterior pole and may cross the avascular foveal region, are seen rarely.

Discussion: Most of these cases are unilateral and stable with excellent visual prognosis and are detected on routine examination. Foveal cysts, hemorrhages and displaced foveola may also be seen.

These entities must be distinguished from racemose angiomas, capillary hemangiomas of the retina and even when associated with neurological symptoms, should be considered as part of the Wyburn-Masson syndrome (Arch Soc Esp Oftalmol 2008; 83: 273276).
\end{abstract}

Key words: Congenital retinal macrovessels, aberrant retinal vessels, displaced fovea.

\section{INTRODUCCIÓN}

Los macrovasos congénitos retinianos son vasos llamativos y estables que cruzan la zona avascular foveal. Se presentan tres casos encontrados de forma casual y sus hallazgos angiográficos característicos.

\section{CASOS CLÍNICOS}

Paciente de 17 años que acude por miodesopsias en ojo derecho (OD) con agudeza visual sin corrección (AVSC) de 1 en ambos ojos (AO). En el fondo de ojo derecho se aprecia una vénula temporal

\footnotetext{
Recibido: 24/10/06. Aceptado: 18/2/08.

Servicio de Oftalmología. Hospital de Cabueñes. Gijón. Principado de Asturias. España.

1 Licenciado en Medicina.

2 Doctor en Medicina.

Comunicación presentada en el LXXXII Congreso de la S.E.O. (A Coruña 2006).

Correspondencia:

Gema Pérez Carro

C/. Velázquez, 2, 2. ${ }^{\circ} \mathrm{I}$

33211 Gijón

España

E-mail: gemurri27@yahoo.es
} 
superior que atraviesa la mácula, siendo normal el ojo izquierdo (OI) (fig. 1).

En la angiografía con fluoresceína (AFG) destacamos: Relleno precoz de la vénula aberrante (fig. 2a), alteración inespecífica de la vénula y del árbol vascular perifoveolar sin dilataciones ni fugas con una zona avascular foveal (ZAF) normal (fig. 2b), probables comunicaciones arteriovenosas y un retraso en vaciamiento de la vénula en fases tardías (fig. 2c). Todas las pruebas complementarias como la rejilla de Amsler, test de los colores y estudio electrofisiológicos son normales así como la campimetría (Humphrey 10-2) sin alteración en la sensibilidad foveal, a pesar de la subjetiva disminución de sensibilidad a la luz. Permanece estable tras 8 meses.

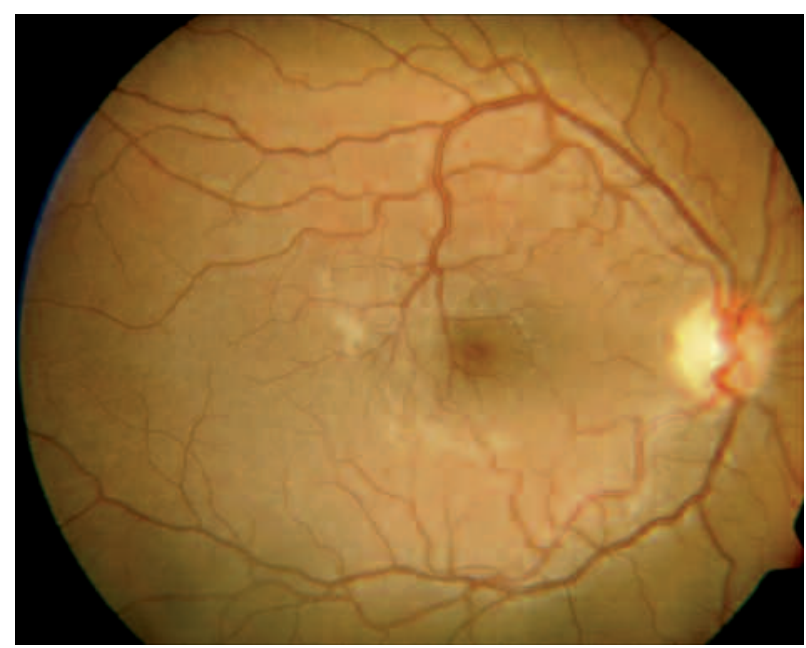

Fig. 1: Vénula temporal superior que se dirige verticalmente a mácula delimitando su lado temporal, cruzando el rafe medio. Caso 1. ${ }^{\circ}$.
Paciente de 48 años que acude por crisis hipertensiva en el que se realiza una exploración oftalmológica para descartar repercusión orgánica. En el fondo de ojo derecho se observa un quiste foveolar y una vénula temporal superior duplicada atravesando la mácula (fig. 3) y en el ojo izquierdo hallazgos compatibles con un síndrome vascular hipertono-hipertensivo grado I.

La AVSC en OD es 0,8 y de 1 en su OI. Las pruebas complementarias son normales.

En la AFG del OD encontramos un relleno pre$\mathrm{coz}$ de la vénula temporal aberrante, una ZAF «dividida en dos» (fig. 4a), focos de hiperfluorescencia maculares, compatibles con imagen de retinografía y con la visión, sin fugas tardías (fig. 4b) y retraso en vaciado de la vénula (fig. 4c). Permanece estable tras 1 año.

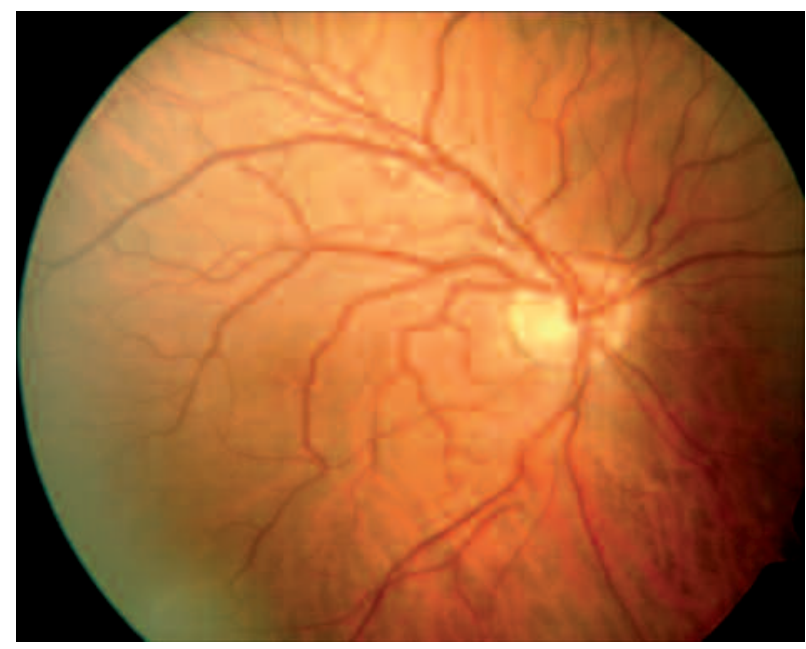

Fig. 3: Quiste foveolar y una vénula temporal superior duplicada y aberrante atraviesa la mácula. Caso 2. .

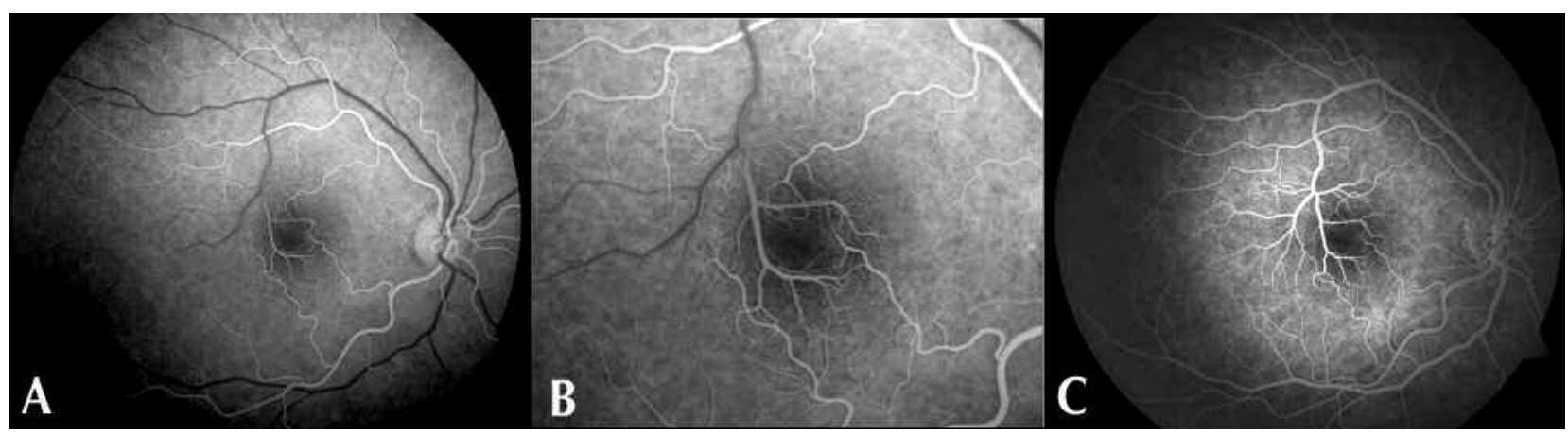

Fig. 2: AFG. A: Fases precoces arteriales, relleno precoz de la vénula aberrante. B: Alteración inespecífica de la vénula aberrante y del árbol vascular perifoveolar sin dilataciones ni fugas con ZAF normal, probables comunicaciones arteriovenosas. C: Retraso en vaciamiento de la vénula en fases tardías. 


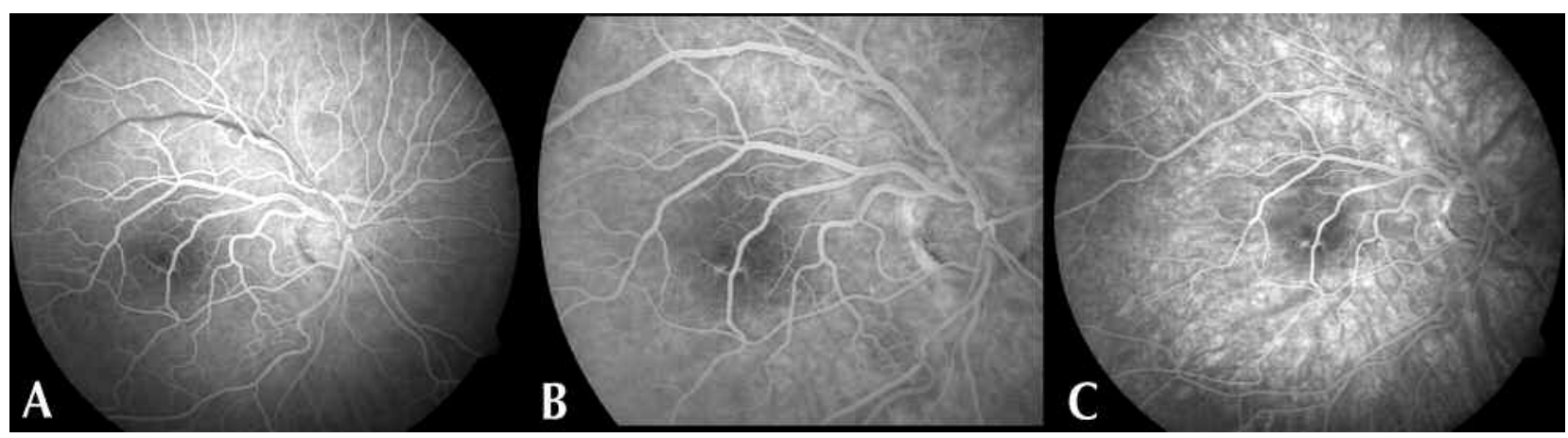

Fig. 4: AFG: A: Relleno precoz de la vénula temporal superior aberrante, una ZAF «dividida en dos». B: Focos de hiperfluorescencia maculares sin fugas tardías. C: Retraso en vaciado de la vénula.

Paciente de 33 años que acude de urgencias por visión borrosa en su ojo derecho. Su AVSC es de 1 difícil en OD y 1 en OI. En el fondo de ojo derecho se aprecia una vénula temporal inferior, que cruza el rafe medio atravesando la fóvea y pérdida de reflejo foveolar (fig. 5) siendo normal el OI. La AFG presenta los mismos hallazgos comentados (fig. 6a y 6b) y las pruebas complementarias son normales.

\section{DISCUSIÓN}

Los macrovasos congénitos retinianos (MCR) son lesiones descritos por Mauthner por primera vez en 1869 y definidos en 1982 por Brown como vasos grandes aberrantes, que cruzan el rafe medio horizontal asintomáticos o asociados a cambios

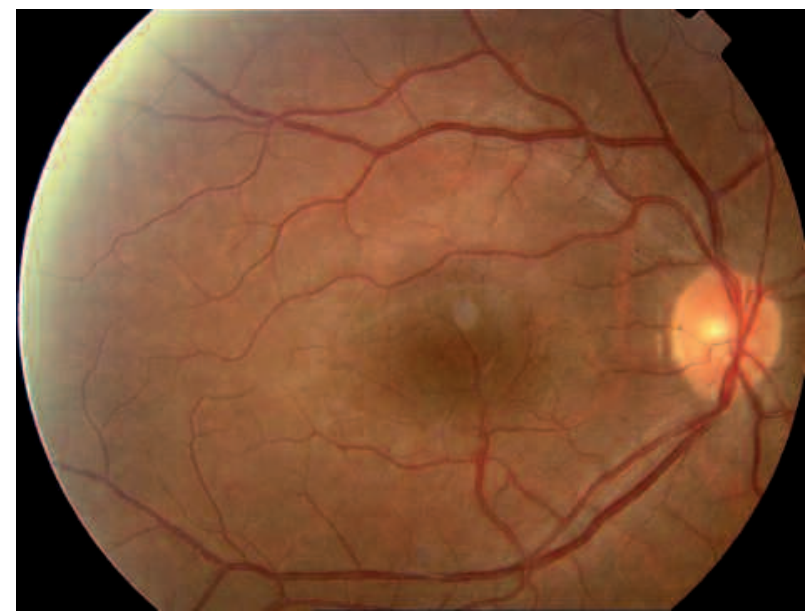

Fig. 5: Vénula Temporal Inferior que se dirige hacia arriba atravesando la fóvea. Pérdida de reflejo foveolar. Caso 3. .
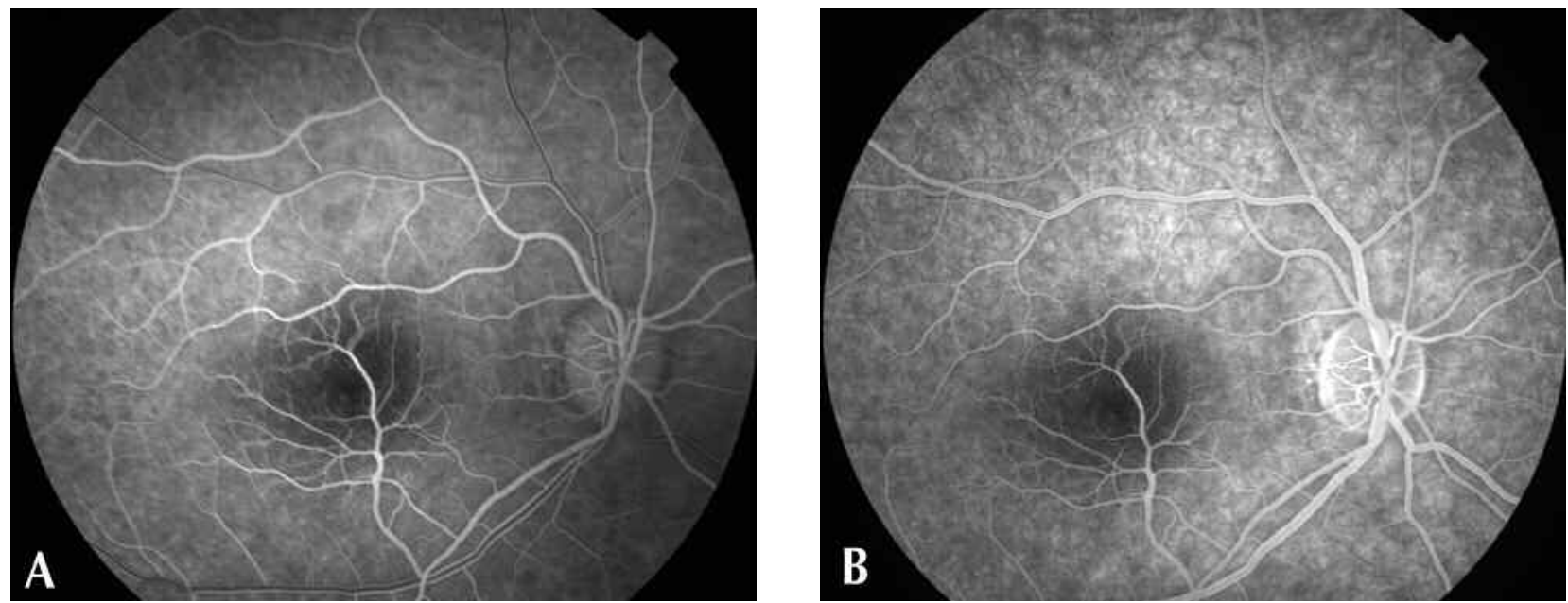

Fig. 6: AFG: A: Relleno precoz de la vénula en fases arteriales. B: Permanencia del contraste en vénula aberrante en fases tisulares. 
mínimos en la visión o en la percepción de los colores (1-3).

Son típicamente unilaterales, por lo general un vénula, que drena o suple a la vez el territorio de ambas hemiretinas, y que de forma aberrante cruzan el polo posterior o la misma fóvea.

Para algunos autores su prevalencia estaría en torno a $1 / 200.000$ (1) y se cree que se forman durante la 15-16 semana de gestación aunque su causa desencadenante se desconoce.

En su mayoría son lesiones identificables hallados en exploraciones de rutina sin embargo debe realizarse un diagnóstico diferencial con otras entidades vasculares como las comunicaciones arteriovenosas, angiomas racemosos, hemangiomas capilares de la retina, bucles vasculares prepapilares, tortuosidad venosa congénita o secundaria a obstrucción venosa (1) e incluso con tumores como el retinoblastoma y melanomas coroideos $(2,3)$.

A este respecto es importante si existen síntomas neurológicos realizar estudios de neuroimagen para descartar la asociación de anomalías vasculares cerebrales como en el síndrome de Wyburn-Masson (1-3).

En ocasiones la anomalía se describe con variaciones con afectación de la visión por ectopia foveolar, reflejo foveal disminuido (3) debidos a cambios en epitelio pigmentario foveal $(1,3)$, quistes foveales y/o hemorragias post-valsalva (1), o al propio trascurso de la vena por la zona avascular foveal como en nuestros casos.

Como hallazgos angiográficos característicos se describen: relleno precoz y vaciamiento retrasado de la vénula, plexo capilar circundante dilatado (4), áreas de no perfusión capilar, hiperfluorescencia por alteraciones en EPR (1), fugas o alteraciones en la pared vascular inespecíficas e incluso la asociación a una comunicación arterio venosa $(1,2,4)$ ya mencionados en nuestros casos.

Algunos autores han descrito alteraciones en la rejilla de Amsler y en la sensibilidad foveal en el campo visual (5) y aunque nosotros hemos encontrado una subjetiva disminución a la sensibilidad luminosa no pudimos demostrarlo con las pruebas complementarias estándares realizadas. La explicación a este fenómeno o angioescotoma estaría en relación con la presencia de hemoglobina circulante en el vaso (5).

En conclusión los MCR o vasos aberrantes son hallazgos llamativos, incidentales, que requieren diagnóstico diferencial con otras entidades retinianas y que, salvo raras excepciones, no ocasionan ninguna alteración para la visión del paciente.

\section{BIBLIOGRAFÍA}

1. Gurwood SA, Bailey JT, Pelino JC. Congenital retinal macrovessel: A case report. Optometry 2001; 72: 597602.

2. Brown CG, Donoso LA, Magargal LE, Goldberg RE, Sarin L K. Congenital retinal macrovessels. Arch Ophthalmol 1982; 100: 1430-1436.

3. Chronister LC, Nyman NN, Meccariello AF. Congenital retinal macrovessel. Optom Vis Sci 1991; 68: 747-749.

4. Polk TD, Park D, Sindt CW, Heffron ET. Congenital retinal macrovessel. Arch Ophthalmol 1997; 115: 290-291.

5. Shah VA, Chalam KV. Congenital retinal macrovessel causes reduced retinal sensitivity at the macula. Eur Ophthalmol 2004; 14: 341-344. 